\title{
The Behaviour of Vertical Bell Laboratories Layered Space-Time Algorithm Combined with Multiuser Detection Schemes in Wireless Communication System
}

\author{
Ayodeji James Bamisaye $^{{ }^{*}}$, Olugbenga Kayode Ogidan ${ }^{2}$, Babatope Osalope ${ }^{1}$ \\ ${ }^{1}$ Department of Switch Technology, Starcomms Telecommunications, Lagos, Nigeria \\ ${ }^{2}$ Information Communications Unit, Ondo State University of Science and Technology, \\ Okitipupa, Nigeria \\ E-mail:ayobamisaye@yahoo.com,gbengaogidan@yahoo.com,topeos@starcomms.com \\ Received November 4, 2010; revised December 5, 2010; accepted January 7, 2011
}

\begin{abstract}
This paper provides the performance analysis of multiuser Vertical Bell Laboratories Layered Space-Time (V-BLAST) system receiver structures for Multiple-input Multiple-Output (MIMO) channel at a base station with assumption of perfect channel estimation and perfect timing delay estimation. In MIMO channels the receivers such as decorrelator, Minimum Mean Square Error (MMSE) and Multistage Parallel Interference Cancellation (MPIC) receiver outperform the conventional receiver. Withal, since the multiple antenna interference led to a strong impact on the performance degradation of a multistage interference cancellation receiver, the performance of MPIC receiver was highly degraded based on system loading.
\end{abstract}

Keywords: CDMA, Multiple-Input Multiple-Output (MIMO) Channel, Multiuser Detection, Vertical Bell Laboratories Layered Space-Time (V-BLAST)

\section{Introduction}

Several techniques are used in wireless communication system for sharing available spectrum resources. These techniques include: frequency division multiple access (FDMA), time division multiple access (TDMA) and code division multiple access (CDMA). (FDMA) assign different frequency to each user, TDMA), assigns different time slots to users. However, the CDMA technique shares the entire bandwidth by distinguishing signals with a unique signature for each user. A promising technique to achieve improved capacity for CDMA is multi-user detection [1].

Recent research on wireless communication systems has shown that using multiple antennas at both transmitter and receiver offers the possibility of communications at higher data rates compared to single antenna systems $[2,3]$. Multi input and multi output (MIMO) system has proved in the recent past to provide very high capacity without any increase in the transmission bandwidth and power. The information-theoretic capacity of these multipleinput multiple-output channels was shown to grow linearly with smaller numbers of transmit and receive antennas in rich scattering environments, and at sufficiently high signal-to-noise (SNR) ratios [4,5].

The performance of multiuser detection along a MIMO downlink system is presented in [6]. A system to enhance signal-to-noise plus noise ratio for Multiple-Input MultipleOutput Code Division Multiple Access (MIMO CDMA) communications in the downlink for frequency-selective fading environments is considered in [7]. The performance of the V-BLAST algorithm combined with multi-user detection in downlink system is evaluated in [8].

MIMO profile in any wire-less communication system can be realized by two schemes namely, 1) using the classical BELL labs architecture (VBLAST-Vertical Bell Laboratories Space Time architecture) and (2) Space Time Block Codes. And OFDM techniques can compensate for multi-path fading effects, which cause time dispersion, and out of band emission of the received signal Of late, significant progress has been made in multiuser detection for code- division multiple-access (CDMA) systems [3]. Conventional correlation receivers fail to realize the full potential benefits of CDMA because they treat Multiple Access Interference (MAI) which is inherent in CDMA, as if it were additive noise. Also, if an interferer is significantly stronger than the desired user, it will dominate performance in a conventional receiver 
because of the near-far problem. To ameliorate this problem stringent power control is required in current CDMA system designs. Thus, CDMA performance can be greatly enhanced by multiuser receivers which compensate for MAI. One of the first investigations into multiuser reception of CDMA signals was presented in [9].

In this paper, we focus on the V-BLAST system combined with multiuser detection schemes without channel coding. The structure of a downlink system is much simpler since the MIMO channel is shared to all users. In this downlink system, we can recover all users' signals by applying various multiuser diction schemes only after equalizing the shared channel. However, for the reverse link, the structure of receivers is more complicated since the channels vary between users [10]. This paper is arranged as follows. Section 2 introduces the system model, Section 3 provides linear and non-linear receiver structures and formulates a detecting algorithm. Simulation results are presented and analyzed in Section 4. Eventually conclusions are drawn in Section 5 .

\section{System Model}

The received baseband signal from user $k$ is defined as a binary phase modulated waveform:

$$
\begin{gathered}
s_{k}(t)=\sqrt{P_{k}} a_{k}(t) b_{k}(t) e^{j \theta_{k}} \\
s_{k}\left(t-\tau_{k}\right)=\sqrt{P_{k}} a_{k}\left(t-\tau_{k}\right) b_{k}\left(t-\tau_{k}\right) e^{j \theta_{k}}
\end{gathered}
$$

Where $P_{k}$ is the $k$ th user's received signal power, $a_{k}$ and $b_{k}$ are the spreading and data waveforms respectively (we assume rectangular pulses for both), That is, $a_{k}$ is made to demultiplex input data stream $b_{k}$. This demultiplexing is the encoding scheme for the V-BLAST at the transmitter and $\theta_{k}$ is the received phase of the $k$ th user relative to some reference phase. $\tau_{k}$ is the time delay that models the asynchronous nature of uplink system which needs not to be considered in down link system. Due to the asynchronous nature of the system uplink, the received signal is:

$$
r(t)=\sum_{k-1}^{k} H^{k} s_{k}\left(t-\tau_{k}\right)+n(t)
$$

$H^{k}$ represents channel matrix for MIMO channel fading affecting the $k^{\text {th }}$ user with size $n_{T} \cdot n_{R}$ whose element $h_{i, j}^{k}$ is the complex fading coefficient for the path from transmit antenna $j$ to receive antenna $i$. We assume a single noise source from a common front end. The set of sufficient statistics can be shown to be a set of matched filter outputs $y$ where the filters are matched to each user's spreading code. In terms of the complex envelope, the vector of sufficient statistics is:

$$
y=y_{I} \cos (\Theta)+y_{Q} \sin (\Theta)
$$

where the ith matched filter output of the $k$ th user is the $(i-1) K+k$ th element of the vector $y, \Theta$ is a $K N_{b} \times K N_{b}$ diagonal matrix where the diagonal elements $\theta_{j, j}$ are the phases of the $i$ th bit of the $k$ th user and $j=(i-1) K+k, K$ is the number of users in the system, $K N_{b}$ is the number of bits in the sequence under consideration and the in-phase and quadrature components are defined as:

$$
y I_{(i-1) K+k}=\int_{(i-1) T+\tau_{k}}^{i T+\tau_{k}} r I(t) a_{k}\left(t-\tau_{k}\right) d t
$$

and

$$
y Q_{(i-1) K+k}=\int_{(i-1) T+\tau_{k}}^{i T+\tau_{k}} r Q(t) a_{k}\left(t-\tau_{k}\right) d t
$$

where $r I(t)=\operatorname{Re}[r(t)], r Q(t)=\operatorname{Im}[r(t)]$ and $\tau_{k}$ is the relative delay of the $k$ th user.

Where the received signal is a vector form like:

$$
r(t)=\left[r^{1}(t) r^{2}(t) \ldots r^{n_{R}}(t)\right]^{T}
$$

The superscripts of elements of the received signal vector represent the $n_{R}^{\text {th }}$ receive antennas. These fading coefficients are modeled as an independent complex Gaussian random variable with zero mean and variance 0.5 per dimension. Figure 1 illustrates the system.

\section{Multiuser Receivers}

\subsection{Conventional Receiver}

The receiver structure is made up of a matched filter bank at each antenna point followed by the V-BLAST decoding block. The outputs of the matched filter banks are rearranged in the form of vector for each corresponding user. In matrix form we represent the set of matched filter outputs as:

$$
y_{I}=R W \cos (\Theta) b+n_{I}
$$

and

$$
y_{Q}=R W \sin (\Theta) b+n_{Q}
$$

where $R$ is a $K N_{b} \times K N_{b}$ matrix

$$
R=\left[\begin{array}{lllll}
H(0) & H(1) & 0 & \cdots & 0 \\
H(-) 1 & H(0) & H(1) & & \vdots \\
0 & H(-1) & H(0) & \ddots & \\
\vdots & \vdots & \vdots & \ddots & H(1) \\
0 & \cdots & 0 & H(-1) & H(0)
\end{array}\right]
$$

The $(k, 1)$ th element of the $K \times K$ matrix $H(i)$ is defined by:

$$
h_{k, l}(i)=\int_{-\infty}^{\infty} a_{k}\left(t-\tau_{k}\right) a_{l}\left(t+i T-\tau_{l}\right) d t
$$

$T$ is the symbol duration, $W$ is a $K N_{b} \times K N_{b}$ diagonal 
matrix of the square root of user received energies defined similar to $\Theta$, b is a $K N_{b}$ length vector with the $j=(i-1) K+k t h$ element equal to the $i$ th data symbol of the $k$ th user, and $n_{I}$ and $n_{Q}$ are vectors of colored noise samples at the matched filter outputs. If the users are numbered such that $\tau_{1}<\tau_{2}<\ldots . \tau_{K}$ then $H(1)$ will be an upper triangular matrix with zeros along the diagonal, $H(-1)=H^{T}(-1)$ and $H(i)=0 \forall|i|>1 . N_{f}$ is the number of consecutive symbols and $K$ is the total number of users loaded in the system.

$\boldsymbol{f}^{i}$ is the $K N_{f}$ length vector form of all users' data symbols transmitted over the MIMO channel described in Equation (11). Each element is expressed in Equation (12).

$$
f_{k}^{i}(l)=\sum_{j-1}^{n_{T}} h_{i, j}^{k} b_{j}(l), l=1,2, \cdots, N_{f}
$$

The statistics are manipulated by the V-BLAST decoding algorithm after the matched filter operation. Here, the operator $\mathbb{V}_{G^{k}}$ is defined for convenience as the V-BLAST decoding processing with the nulling matrix $G^{k}$, which represents the pseudo-inverse channel matrix of $k^{\text {th }}$ user. The pseudo-inverse channel matrix is represents by:

$$
\begin{gathered}
G^{k}=\left(H^{k+} H^{k}\right)^{-1} H^{k+} \\
G^{k}=\left(H^{k+} H^{k}+\frac{\sigma_{n}^{2}}{\sigma_{d}^{2}}\right)^{-1} H^{k+}
\end{gathered}
$$

Equation (13) is a pseudo-inverse channel matrix for the ZF nulling criterion and Equation (14) is for the MMSE nulling criterion. This operation cancels the multiple antenna or spatial interference caused by the V-BLAST decoding algorithm in the manner of parallel interference cancellation or serial interference cancellation. Therefore, the recovered symbols are represented by:

$$
b_{k}=\mathbb{V}_{G^{k}}\left(\left[y_{k}^{1} y_{k}^{2} \ldots \ldots y_{k}^{n_{R}}\right]^{T}\right) k=1,2 \ldots, K
$$

Since the correlation matrix contributed to the transmitted symbols even in V-BLAST, the desired signal within a conventional receiver cannot avoid the interference from other user's signals especially in an asynchronous system.

\subsection{Decorrelating Receiver}

In a linear detector, the decision metric is a linear transformation of the sufficient statistics that performs inter- ference cancellation by linear transformation for finding the decision metric i.e.

$$
\boldsymbol{d}=\operatorname{sgn}\left[T y_{I} \cos (\Theta)+T y_{Q} \sin (\Theta)\right]
$$

The decorrelator is a linear detector where $T=R^{-1}$. The decorrelator removes the correlation between the elements of $y[10]$.

$$
\boldsymbol{d}=\operatorname{sgn}\left[W b+R^{-1} n\right]
$$

This transformation is derived from the maximization of the likelihood function or equivalently the minimization of $(y-R b)^{T} R^{-1}(y-R b)[2]$.

The decorrelating receiver is a linear detector. Eliminating the interference from undesired signals at the first stage using:

$$
\boldsymbol{d}^{i}=\left[T y^{i}\right] i=1,2 \cdots n_{R}
$$

The linear transformation, $T=R^{-1}$, is obtained from the maximization of the likelihood function or equivalently the minimization of

$\Lambda(\Theta)=(Y-R \Theta)^{T} R^{-1}(Y-R \Theta)$ where y represents the output of matched filter and $\Theta$ is estimated signal.

$$
\begin{aligned}
\boldsymbol{d}^{i} & =R^{-1} y^{i} i=1,2 \cdots n_{R} \\
& =W b^{i}+R^{-1} n i=1,2 \ldots n_{R}
\end{aligned}
$$

At the second stage, after rearranging the decorrelated signals, the V-BLAST decoding algorithm is applied to the signal $\boldsymbol{d}^{i}$ where the spatial interference of undesired signals is reduced and the symbols are decided as follows:

$$
b_{k}=\mathbb{V}_{G^{k}}\left(\left[\boldsymbol{d}_{k}^{1} \boldsymbol{d}_{k}^{2} \cdots \in \boldsymbol{d}_{k}^{n_{R}}\right]^{T}\right) k=1,2 \cdots, K
$$

\subsection{Minimum Mean Square Error (MMSE) Receiver}

A similar receiver structure can be obtained if the transformation is sought which minimizes the mean square error of the bit estimate, $\left[(b-y)^{T}(b-y)\right]$. In this case the linear transformation $T=R^{-1}$ used in [10] $\boldsymbol{d}=\operatorname{sgn}\left[W b+R^{-1} n\right]$ is replaced by $T=\left(R+\frac{N_{0}}{2 W^{2}}\right)^{-2}$.

The performance of the MMSE detector approaches the decorrelator as $N_{0} \rightarrow 0$. As $N_{0}$ grows large, T approaches an identity matrix scaled by $N_{0} / 2$ and is thus reduced to the conventional receiver. Thus, the MMSE detector seeks to strike a balance between removing the interference and not enhancing the noise. At low

$$
\begin{aligned}
& \boldsymbol{f}^{i}=\left[\boldsymbol{f}_{1}^{i}(1) \boldsymbol{f}_{2}^{i}(1) \cdots \boldsymbol{f}_{k}^{i}(1) \boldsymbol{f}_{1}^{i}(2) \boldsymbol{f}_{2}^{i}(2) \cdots \boldsymbol{f}_{k}^{i} \boldsymbol{f}_{1}^{i}\left(N_{f}\right) \boldsymbol{f}_{2}^{i}\left(N_{f}\right) \cdots \boldsymbol{f}_{k}^{i}\left(N_{f}\right)\right]^{\mathrm{T}} \\
& \boldsymbol{d}=\left[\boldsymbol{d}_{1}^{i}(1) \boldsymbol{d}_{2}^{i}(1) \cdots \boldsymbol{d}(1) \boldsymbol{d}_{1}^{i}(2) \boldsymbol{d}_{2}^{i}(2) \cdots \boldsymbol{d}_{k}^{i} \boldsymbol{d}_{1}^{i}\left(N_{f}\right) \boldsymbol{d}_{2}^{i}\left(N_{f}\right) \cdots \boldsymbol{d}_{k}^{i}\left(N_{f}\right)\right]^{T}
\end{aligned}
$$




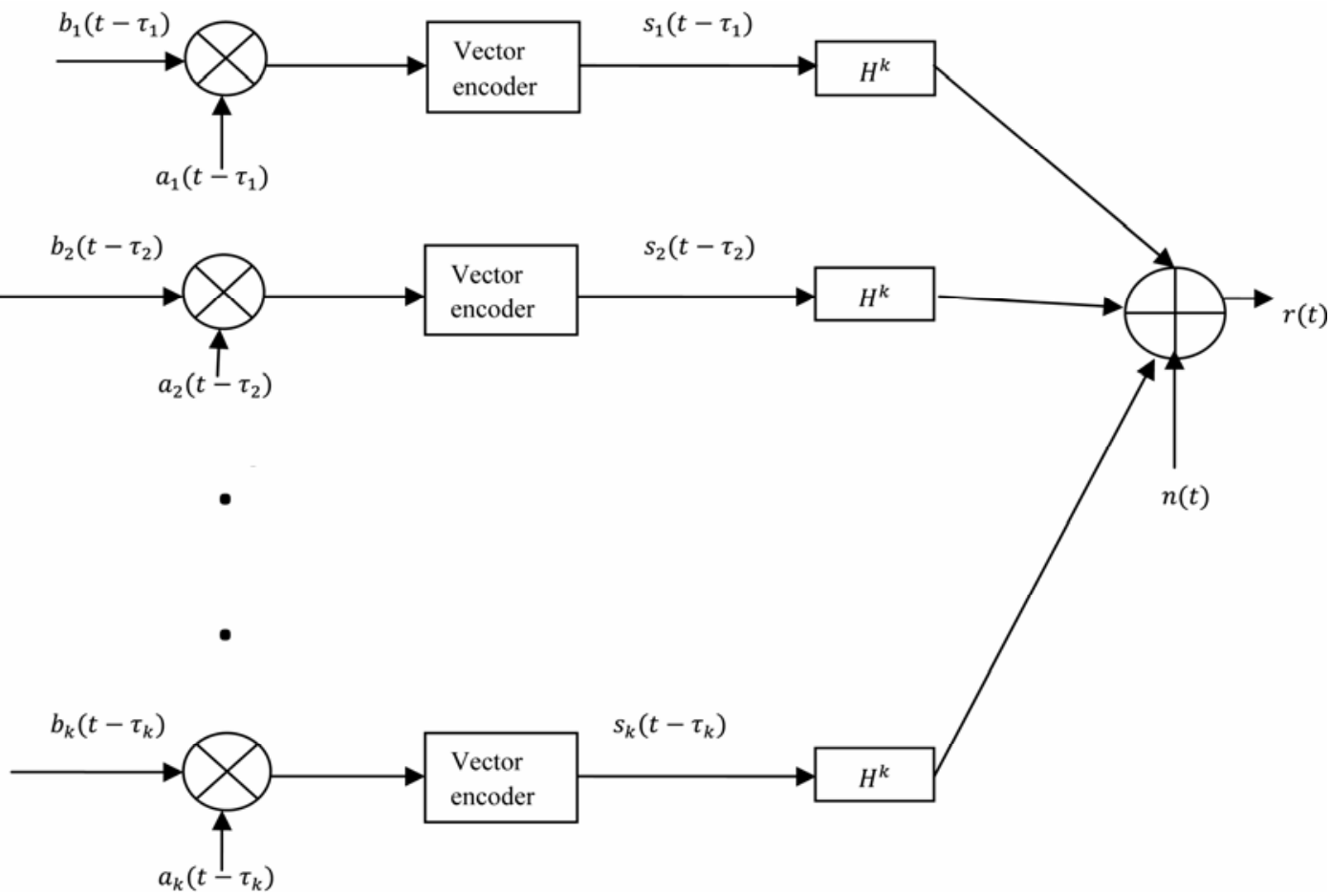

Figure 1. Model of multiuser v-blast system on mimo channel.

$E_{b} / N_{0}$ the MMSE receiver will outperform the décor relator, while the decorrelator has superior BER per formance at high $E_{b} / N_{0}$. Due to the residual interference this transformation results in an estimate which is biased, and its performance is dependent on the power levels of the interferers.

$$
\boldsymbol{d}^{i}=\left(R+\frac{N_{0}}{2 W^{2}}\right)^{-1} y^{i} i=1,2 \ldots n_{R}
$$

As a spatial interference cancellation step, the VBLAST decoding block is the same as for the decorrelator by:

$$
b_{k}=\mathbb{V}_{G^{k}}\left(\left[\boldsymbol{d}_{k}^{1} \boldsymbol{d}_{k}^{2} \cdots \boldsymbol{d}_{k}^{n_{R}}\right]^{T}\right) k=1,2 \cdots, K
$$

\subsection{Multistage Parallel Interference Cancellation (MPIC)}

Multistage receivers are receivers which have multiple stages of interference estimation and cancellation. This cancellation approach was developed in $[11,12]$. In [11] it was suggested that each user's signal could be iteratively estimated in parallel. The estimates for each user can then be used to reduce the interference by subtracting the estimate of each interferer from the desired user's signal. The entire process can be repeated for several stages. At each stage, better estimates of each user are produced, allowing more effective interference cancellation. In this paper we assume the use of matched filters at each stage for estimation. This allows a single estimate (the matched filter output) to be used for both the data symbol and the channel gain and alleviates the need for any outside power estimates. Mathematically we can represent the decision metric for an S-stage parallel cancellation scheme as:

$$
b_{k}=\mathbb{V}_{G^{k}}\left(\left[y_{k}^{1(S)} y_{k}^{2(S)} \cdots \cdots y_{k}^{n_{R(S)}}\right]^{T}\right) k=1,2 \cdots, K
$$

where

$$
\begin{gathered}
y_{k}^{i(s)}=\frac{1}{T} \int_{(i-1) T+\tau_{K}}^{i T+\tau_{k}} \boldsymbol{\tau}_{k}^{i(s)}(t) a_{k}\left(t-\tau_{k}\right) d t, i=1,2 \cdots n_{R}, k=1,2 \cdots, K \\
\boldsymbol{\tau}_{k}^{i(s)}(t)=r(t)^{i}-\sum_{j \neq k} y_{j}^{s-1} a_{j}\left(t-\tau_{j}\right)
\end{gathered}
$$

$j=(i-1) K+k$, for the $i$ th bit of the $k$ th user and $\boldsymbol{r}_{k}^{i(s)}(t)$ is the $k$ th user's in-phase after s-1 stages of cancellation.

$$
y_{k}^{i,(s)}=r(t)^{i}-\sum_{j \neq k} y_{j}^{(s-1)}(t) a_{j}\left(t-\tau_{j}\right) d t, i=1,2 \ldots . n_{R}, k=1,2 \ldots \ldots, K
$$


The superscript $i$ represents the receive antenna number, $k$ is the user number, $r_{k}^{i,(s)}(t)$ represents the Sstage signal of the $k^{\text {th }}$ user at antenna $i$ after cancellation, $a_{k}$ represents the spreading sequence of the $k^{\text {th }}$ user, and $\tau_{k}$ represents the estimated time delay of the $k^{\text {th }}$ user. The operator $\mathbb{V}_{G^{k}}$ represents the V-BLAST decoding processing. A bias reduction technique for multistage interference cancellation is verified in [1]. The bias increases linearly with system loading such that the bias influences the decision statistics in the first stage of multiple access interference cancellation. The effect of bias is mitigated for subsequent stages. To reduce the effect of bias, a partial cancellation factor $C^{(S)}$ is employed. This factor varies at every stage in the range 0,1 . The partial-cancellation factor $C^{(S)}$ of 0.5 at second stage gives good performance improvement [8]. The mathematical form of cancellation with the partial cancellation factor is formulated by Equation (29) as below.

\section{Simulation Results and Analysis}

The ZF nulling V-BLAST algorithm based on successive spatial interference cancellation is employed. Perfect power control, perfect channel estimation and time delay estimation are assumed. Rayleigh flat fading channel is considered. We define one frame as one packet of 20 symbols. The channel coefficients are modeled with an independent zero mean complex Gaussian random variable with variance 0.5 per dimension. The channel coefficients are constant during one frame transmission. Gold sequences with 31 processing gain are utilized. Each chip of the spreading sequences is sampled at 5 samples/chip. Timing delays are generated randomly to realize an asynchronous model.

Figure 2 depicts the performance of three-stage PIC with the various partial-cancellation factors at second stage. For the lightly loaded system, partial cancellation factors of 0.7 and 0.9 give good reduction of the multiple access interference at the second stage. However, partial-cancellation factors of 0.7 and 0.5 mitigate the effect of bias relatively well for a highly loaded system. By comparison, the partial-cancellation factor 0.7 can be considered as the best choice at flat fading MIMO channel. The capacity curves for $E_{b} / N_{0}=20 \mathrm{~dB}$ and processing gain $N=31$ are shown in Figure 3.

It was observed that the performance of the decorrelator, MMSE detector and two-stage PIC combined with decorrelator at first stage are equivalent. More so, they exhibit enormous performance improvement compared to the conventional receiver. However, the behaviour of the three-stage (parallel interference cancellation) PIC receiver, that is a non-linear interference cancellation receiver, gradually degrades as the number of active users increase even though it outperforms the conventional receiver.

Figure 4 illustrates results for a system with 10 users, showing how the number of stages affects the performance improvement for multistage PIC. It shows how the number of stages affects the performance improvement of multistage PIC. It was observed that the performance of MPIC is improved as the number of stages increases.

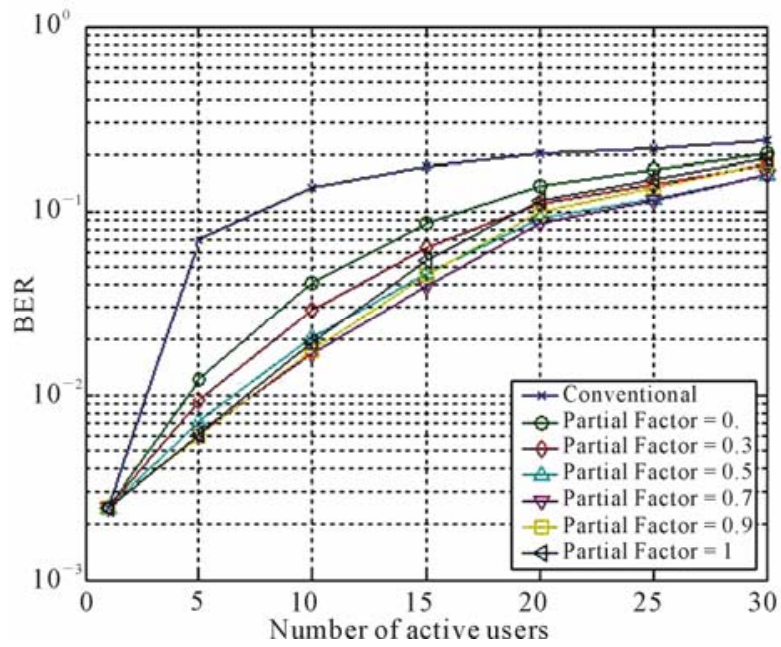

Figure 2. BER versus number of active users as influenced by partial-cancellation factor for MPIC in Flat Fading MIMO channels.

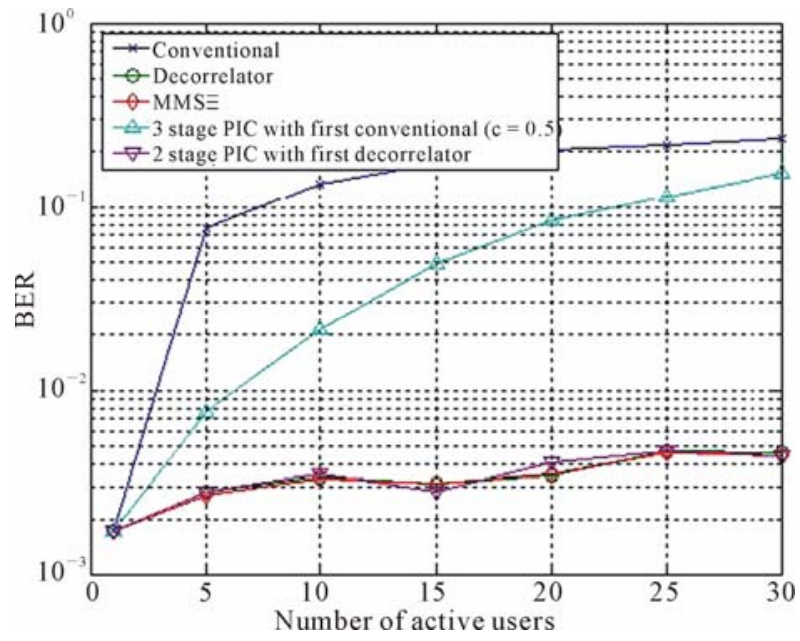

Figure 3. BER versus number of active users with perfect channel estimation in Flat Fading MIMO channels.

$$
r_{k}^{i,(s)}(t)=r(t)^{i}-C^{(s)} \sum_{j \neq k} y_{j}^{(s-1)} a_{j}\left(t-\tau_{j}\right), i=1,2 \ldots n_{R}, k=1,2 \ldots \ldots, K
$$




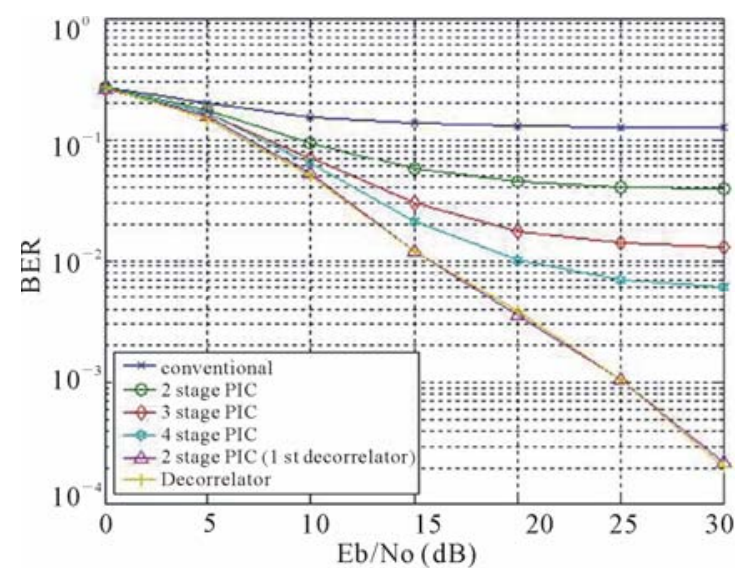

Figure 4. BER versus Eb/No with perfect channel estimation under Flat fading MIMO channels.

However, the MPIC performance does not equal the performance of linear detectors such as the decorrelator and the MMSE receiver since the multiple antenna interference prevents the MPIC receivers from cancelling out the multiple access interference appropriately. We recognize that the multiple antenna interference affects the performance of the non-linear multiple access interference cancellation receiver. Therefore, the MPIC receivers are not suitable without a channel coding scheme for MIMO channels.

\section{Conclusions}

This paper investigated the behaviour of V-BLAST combined with receiver structure of multiuser detectors. The performance of MPIC receivers with various partial-cancellation factors was verified. All receivers such as the decorrelator, MMSE and MPIC achieve the tremendous performance improvement compared to the conventional receiver that does not eliminate multiple access interference. However, the MPIC receiver, which is one of the nonlinear detectors, over the MIMO channels, is not robust to interference from multiple antennas that obstruct cancellation at each stage, whereas linear detectors are not as severely affected by multiple antenna interference.

\section{References}

[1] S. Verdu, "Multiuser Detection," Cambridge University Press, Cambridge, 1998.
[2] N. Prabagarane, M. Ramakrishnan, T. Divya, M. Thomas and B. Jalaja, "Performance Evaluation of Multi Stage Receivers for Coded Signals in MIMO Channels," 4th IEEE WiCOM, October 2008.

[3] V. Arun, A. S. S. Vasan and L. Krishnan, "Investigations on the Performance of MIMO Assisted Multi Carrier DS/CDMA System with Multiuser Detection for 4G Mobile Communications," Project Report, SSN Institutions, 2009.

[4] A. J. Bamisaye and M. O. Kolawole, "Evaluation of Downlink Performance of a Multiple-Cell, Rake Receiver Assisted CDMA Mobile System," Wireless Sensor Network, Vol. 2, No. 1, 2010, pp. 1-6.

[5] A. J. Bamisaye and M. O. Kolawole, "Uplink Performance Evaluation of CDMA Communication System with Rake Receiver and Multiple Access Interference Cancellation," International Journal of Communications, Networks and System Science, Vol. 3, No. 6, 2010, pp. 540-547.

[6] H. Dai and A. F. Molisch, "Multiuser Detection for Interference-Limited MIMO Systems," 55th IEEE Vehicular Technology Conference, Vol. 1, Spring 2002, pp. 4549.

[7] R. L. Choi, R. D. Murch and K. B. Letaief, "MIMO CDMA Antenna System for SINR Enhancement," IEEE Transactions on Wireless Communications, Vol. 2, No. 2, March 2003, pp. 240-249.

doi:10.1109/TWC.2003.808961

[8] D. Samardzija, P. Wolniansky and J. Ling, "Performance Evaluation of the VBLAST Algorithm in W-CDMA Systems," IEEE Vehicular Technology Conference, Vol. 2, Fall 2001, pp. 723-727.

[9] K. S. Schneider, "Optimum Detection of Code Division Multiplexed Signals," IEEE Transactions on Aerospace and Electronic Systems, Vol. AES-15, No. 1, January 1979, pp. 181-185. doi:10.1109/TAES.1979.308816

[10] W. Wu and K. Chen, "Linear Multiuser Detectors for Synchronous CDMA Communication over Rayleigh Fading Channels," 17th IEEE International Symposium, Personal, Indoor and Mobile Radio Communications, PIMRC'96, Vol. 2, October 1996, pp. 578-582.

[11] M. K. Varanasi and B. Aazhang, "Multistage Detection in Asynchronous Code-Division Multiple Access Communications," IEEE Transaction Communication, Vol. 38, April 1990, pp. 509-519. doi:10.1109/26.52662

[12] R. Kohno, H. Imai, M. Hatori and S. Pasupathy, "An Adaptive Canceller of Cochannel Interference for SpreadSpectrum Multiple-Access Communication Networks in a Power Line," IEEE Journal on Selected Areas in Communications, Vol. 8, No. 4, May 1990, pp. 691-699. doi:10.1109/49.54465 